\title{
Population synthesis on ultra-luminous X-ray sources with an accreting neutron star: Wind Roche-lobe overflow cases
}

\author{
Zhao-Yu Zuo, Hao-Tian Song, and Han-Chen Xue
}

\author{
School of Physics, Xi'an Jiaotong University, Xi' an 710049, PR China \\ e-mail: zuozyu@xjtu.edu.cn
}

Received 12 March 2021 / Accepted 16 April 2021

\begin{abstract}
Very recently, wind Roche-lobe overflow (WRLOF) has been suggested as a possible mass transfer mechanism for ultra-luminous X-ray sources (ULXs) and, to date, two neutron-star (NS) ULXs (i.e., NGC 7793 P13 and NGC 300 ULX-1) are remarkable and hard to understand in the current, usual RLOF picture. In this work, we test if the two sources could fit into the WRLOF paradigm. By using an evolutionary population synthesis method, we modeled the population of NS ULXs with (super)giant donors, taking the WRLOF accretion mode into account. We find that the population of wind-fed NS ULXs in the WRLOF mode is distinct in numbers and binary parameters from that in the traditional Bondi-Hoyle-Lyttleton mode, and it is strongly metallicity dependent. The number of NS ULXs with (super)giant donors can be enhanced greatly, by one or two orders of magnitude, depending on the metallicity adopted. Sources with massive $\left(\sim 15-40 M_{\odot}\right)$ (super)giant donors dominate wind-fed NS ULXs in the very low metallicities, while sources in near solar cases are dominated by a red supergiant with a lower mass $M_{2}<10 M_{\odot}$ instead. Moreover, the two NS ULXs can be well reproduced in the WRLOF paradigm, which significantly enriches our understanding of the nature of ULXs and the population. We also present the current distributions of binary parameters of wind-fed NS ULXs, which may be further testified by future high-resolution optical and X-ray observations of these populations.
\end{abstract}

Key words. stars: evolution - X-rays: binaries - stars: neutron - methods: statistical - binaries: close

\section{Introduction}

Ultraluminous X-ray sources (ULXs) are non-nuclear, point-like objects with apparent luminosities of $L_{X}>10^{39} \mathrm{erg} \mathrm{s}^{-1}$, exceeding the Eddington limit for a $10 M_{\odot}$ black hole $(\mathrm{BH}$, Fabbiano 1989, see Kaaret et al. 2017 for a recent review). Although suggested to be intermediate mass $\left(10^{2}-10^{5} M_{\odot}\right)$ BHs (Colbert \& Mushotzky 1999) in early studies as the accretor in ULXs, growing pieces of evidence (Gladstone et al. 2009; Liu et al. 2013; Sutton et al. 2013; Walton et al. 2018) demonstrate that most if not all ULXs are instead stellar-mass binary systems, proposed due to super-Eddington accretion (Begelman 2002) and/or geometric beaming (King et al. 2001; Poutanen et al. 2007; King 2008; King \& Lasota 2020). Strikingly, ULXs can also harbor an accreting neutron star (NS), of which the Eddington limit $L_{\text {Edd }}$ is much lower (i.e., around $2 \times 10^{38} \mathrm{erg} \mathrm{s}^{-1}$ for a $1.4 M_{\odot}$ $\mathrm{NS}^{1}$ ). Several NS ULXs have been identified so far and they are characterized by regular pulses with periods of $\sim 1 \mathrm{~s}$, that is, M 82 X-2 (Bachetti et al. 2014), NGC 7793 P13 (Fürst et al. 2016, 2018; Israel et al. 2017a; Motch et al. 2014), NGC 5907 ULX1 (Israel et al. 2017b), NGC 300 ULX1 (Carpano et al. 2018; Heida et al. 2019b), NGC 1313 ULX-2 (Sathyaprakash et al. 2019), and M 51 ULX-7 (Rodríguez Castillo et al. 2020), or a cyclotron resonance feature, for example, M51 ULX8 (Brightman et al. 2018)

\footnotetext{
1 In the case of a strong magnetic field, the effective Eddington luminosity of NS can be increased by a factor of $\left(\mu_{\mathrm{B}} / \mu\right)^{2}$ for frequency $\mu$ below cyclotron $\left(\mu_{\mathrm{B}}\right)$ due to the reduction of the electron-scattering cross-section (Dall'Osso et al. 2016; Ekşi et al. 2015; Tong 2015).
}

In order to account for the ultra-high luminosity of NS ULXs, mass transfer through Roche-lobe overflow (RLOF) via an accretion disk has always been assumed in previous theoretical studies (Shao \& Li 2015; Fragos et al. 2015; Wiktorowicz et al. 2015, 2017, 2019), as a highly superEddington mass transfer rate is unlikely to reach through stellar wind. For example, after the discovery of the first NS ULX (i.e., M 82 X-2, Bachetti et al. 2014), Shao \& Li (2015) first studied the population of NS ULXs by the use of both evolutionary population synthesis (EPS) and a detailed binary evolution method. They suggest that NS ULXs may contribute significantly to the whole ULX population. And the distribution of the NS ULX population was demonstrated in the donor mass-orbital period plane (see their Figs. 3 and 4), as well. Utilizing the StarTrack EPS code, Wiktorowicz et al. (2017) show that the typical donor of NS ULXs is a red giant star with a mass of $\sim 1.0 M_{\odot}$. They found that massive supergiant donors are rare $(<1 \%)$ in their calculations. Interestingly, several massive stars have already been detected in the optical and infrared as potential donors for ULXs (Kaaret et al. 2004; Liu et al. 2004, 2013; Heida et al. 2014, 2015, 2016; Villar et al. 2016; Lau et al. 2019). Among them, two NS ULXs are remarkable and hard to understand in the current, usual RLOF picture (Shao \& Li 2015; Wiktorowicz et al. 2017). One is NGC 7793 P13 (hereafter P13; Motch et al. 2014; Fürst et al. 2018), which is a pulsing ULX with a blue supergiant (B9Ia) of $18-23 M_{\odot}$ in a long orbital period of about $64 \mathrm{~d}$. Another is the pulsing ULX source NGC 300 ULX-1 (Carpano et al. 2018; Heida et al. 2019b), with a red supergiant (RSG) donor $\left(T_{\text {eff }}=3650-3900 \mathrm{~K}\right.$ and $\log \left(L_{\text {bol }} / L_{\odot}\right)=4.25 \pm 0.10$, Heida et al. 2019a). 
Very recently, wind Roche-lobe overflow (WRLOF, first introduced by Podsiadlowski \& Mohamed 2007; Mohamed \& Podsiadlowski 2007 has been suggested as a possible mass transfer mechanism for ULXs (El Mellah et al. 2019a; Heida et al. 2019a,b). In this scenario, WRLOF can remain stable for large mass ratios while still leading, for relatively sufficient slow winds, to the formation of a wind-captured disk around the accretor, even when the donor does not fill its Roche lobe (RL, El Mellah et al. 2019b). El Mellah et al. (2019a) find that the mass-transfer rate can be boosted much higher than normal Bondi-Hoyle-Lyttleton (BHL) wind accretion (see their Fig. 3), necessary to reach the ULX luminosity level. We note several observations also support the idea of the WRLOF regime (Orosz et al. 2011; Liu et al. 2013). However, population synthesis on NS ULXs considering WRLOF is still lacking.

In the present work, we applied an up-to-date EPS code to investigate the population of NS ULXs in the case of the WRLOF scenario. In the EPS code, we implemented the mass transfer efficiencies $\mu$ computed by El Mellah et al. (2019a). We examined several parameters, such as metallicity and the exponent $\beta$ of the velocity law, which may affect the stellar wind and hence the formation and evolution of WRLOF NS ULXs significantly. The objective of this Letter is to see if the two NS ULXs (i.e., P13 and NGC 300 ULX-1) can be explained in the WRLOF scenario within the range of a reasonable value of key parameters. We also explored the detailed components of wind-fed NS ULX populations, which may help understand the nature of the sources and may be testified by future observations.

This Letter is organized as follows. In Sect. 2, we describe the EPS method and the input physics for wind-fed NS ULXs in our model. The calculated results are presented in Sect. 3. We discuss and summarize our results in Sect. 4.

\section{Models}

We used the EPS code initially developed by Hurley et al. (2000, 2002) and updated by Zuo et al. (2014) to simulate the population of wind-fed NS ULXs. Several major updates have been made to this code, including the common envelope (CE) evolution (Xu \& Li 2010; Loveridge et al. 2011), compact object (CO) mass prescription (i.e., the rapid supernova mechanism, Fryer et al. 2012; Belczynski et al. 2016), and natal kicks of CO formation (i.e., fallback prescription, Fryer et al. 2012). For NS formation, we adopted a Maxwellian kick distribution with a dispersion velocity of $\sigma_{\text {kick }}=265 \mathrm{~km} \mathrm{~s}^{-1}$ (Hobbs et al. 2005) for NSs that formed from core-collapse supernova explosion ( $\mathrm{SNe}$ ). We also considered the formation of low mass NSs through electron-capture supernova (i.e., ECS, Podsiadlowski et al. 2004), which typically has small kicks (Dessart et al. 2006, we assumed $\sigma_{\text {kick }}=50 \mathrm{~km} \mathrm{~s}^{-1}$ ). The maximum NS mass was assumed to be $3 M_{\odot}$, above which BH was assumed to form. For CE evolution, we adopted the energy budget approach (Webbink 1984, 2008) and chose $\alpha_{\mathrm{CE}}=0.9$ throughout (Zuo \& Li 2014).

We assumed that all stars were formed in binaries (i.e., the binary fraction $f_{\mathrm{b}}=1$ ) and evolved $8 \times 10^{6}$ primordial binaries in each model. The initial mass function (IMF) of Kroupa et al. (1993) was taken for the primary star, with mass $M_{1} \in$ $[7,60]$ in solar mass. For the mass of the secondary, a flat distribution of the mass ratio $q \equiv M_{2} / M_{1}$ between 0 and 1 was adopted. We assumed that $\ln (a)$ was evenly distributed between $a=3$ and $10^{4} R_{\odot}$. The tidal effect was taken into account to remove any eccentricity induced in a post-SN binary prior to the onset of mass transfer. The star formation rate (SFR) was fixed to be constantly $3 M_{\odot} \mathrm{yr}^{-1}$ over the $13 \mathrm{Gyr}$ period (Milky Way-like).

The stellar wind of massive stars is vital in our simulations. For the wind mass-loss, Vink et al. (2001) winds were applied for hot, massive stars, that is, metal-dependent fitting formulae given by Belczynski et al. (2010, that is to say Eqs. (6) and (7)). For Wolf-Rayet wind, we chose a combination of the Hamann \& Koesterke (1998) wind and Vink \& de Koter (2005) metal-dependent wind (i.e., Eq. (9), Belczynski et al. 2010). We adopted Eq. (8) in Belczynski et al. (2010) for luminous blue variable stars $\left(L>6 \times 10^{5}\right.$ and $10^{-5} R L^{1 / 2}>1.0$, Humphreys $\&$ Davidson 1994), which is independent of metallicity. The other wind parameters are the same as in Hurley et al. (2000) if not mentioned otherwise. Due to the dependance of metallicity which may influence stellar winds significantly, we considered three choices of metallicity, that is, $Z=Z_{\odot}(=0.02), Z=0.2 Z_{\odot}$ and $Z=0.02 Z_{\odot}$ in our models, to see its effects. It is difficult to determine the wind velocity accurately. We set it proportional to the escape velocity from the surface of the mass-losing star, as a ratio $\beta_{\text {wind }}$ (Hurley et al. 2002; Belczynski et al. 2008). The value of $\beta_{\text {wind }}$ depends on the spectral type of the masslosing star (Lamers et al. 1995). We adopted $\beta_{\text {wind }}=7$ for the most massive MS stars $\left(>120 M_{\odot}\right), \beta_{\text {wind }}=0.5$ for low-mass MS stars $\left(<1.4 M_{\odot}\right)$, and interpolated in between. We adopted $\beta_{\text {wind }}=0.125$ (i.e., slow winds) for extended $\left(R_{\text {don }}>900 R_{\odot}\right)$ H-rich giants. For He-rich stars, we adopted $\beta_{\text {wind }}=7$ for $M_{\text {don }}>120 M_{\odot}$ and $\beta_{\text {wind }}=1.3$ for $M_{\text {don }}<10 M_{\odot}$, and interpolated in between. We note the terminal wind velocity of low mass He-rich stars is still very uncertain. The value we adopted here is different from that suggested by Belczynski et al. (2008), which is too low (J. S. Vink, priv. comm.).

For wind accretion, we adopted the commonly used BondiHoyle-Lyttleton (BHL) mass-accretion-rate formula (Bondi \& Hoyle 1944, see Eq. (6) in Hurley et al. 2002) for fast wind cases. When the orbital and wind velocities are comparable, the WRLOF mode was taken into account. In practice, if the mass transfer rate in the WRLOF mode is higher than the BHL rate, we adopted the WRLOF rate, otherwise the BHL rate was kept the same as in Hurley et al. (2002). For the WRLOF mode, we used the fitting formula based on data obtained from numerical simulations by El Mellah et al. (2019a, Fig. 2 and the data tables they provided) to conduct the calculation. In their simulation, the fraction of stellar wind captured, $\mu$, only depends on the mass ratio $q=M_{\mathrm{don}} / M_{\mathrm{CO}}$, the stellar filling factor $f=R_{\mathrm{don}} / R_{\mathrm{RL}}$ (Eggleton 1983), the exponent $\beta$ of the velocity law, and the ratio of the terminal wind speed to the orbital speed $\eta=v_{\infty} / v_{\text {orb }}$, with $v_{\text {orb }}=2 \pi a / P_{\text {orb }}$ and $P_{\text {orb }}$ being the orbital period. Since only two mass ratios for $q(q=15$ for the NS and $q=2$ for the $\mathrm{BH})$ are provided for $\mu$ in their simulations, a linear interpolation (in logarithmic space) between the two extremal points was conducted for other cases than $q=2$ and 15, which is expected to give a reasonable value of $\mu$ (suggested by Ileyk El Mellah). Due to the large uncertainties of the parameter $\beta$, which represents how fast the wind reaches its terminal speed (i.e., the efficiency of the acceleration), we adopted $\beta=1$ (models labeled "B1"), $\beta=2$ (models labeled "B2"), and $\beta=3$ (models labeled "B3") to test its effects. We also designed models with only the BHL mode applied (models labeled "BHL") for comparison with the above WRLOF models (i.e., models B1, B2, and B3). Then combined with the three choices of metallicity, twelve models were constructed in total. Then the final absolute X-ray luminosity released by accretion onto a $\mathrm{CO}$ fed by a stellar companion could be calculated with the traditional formula $L_{\mathrm{X}}=0.1 \dot{M} c^{2}$, without considering the Eddington limit. 

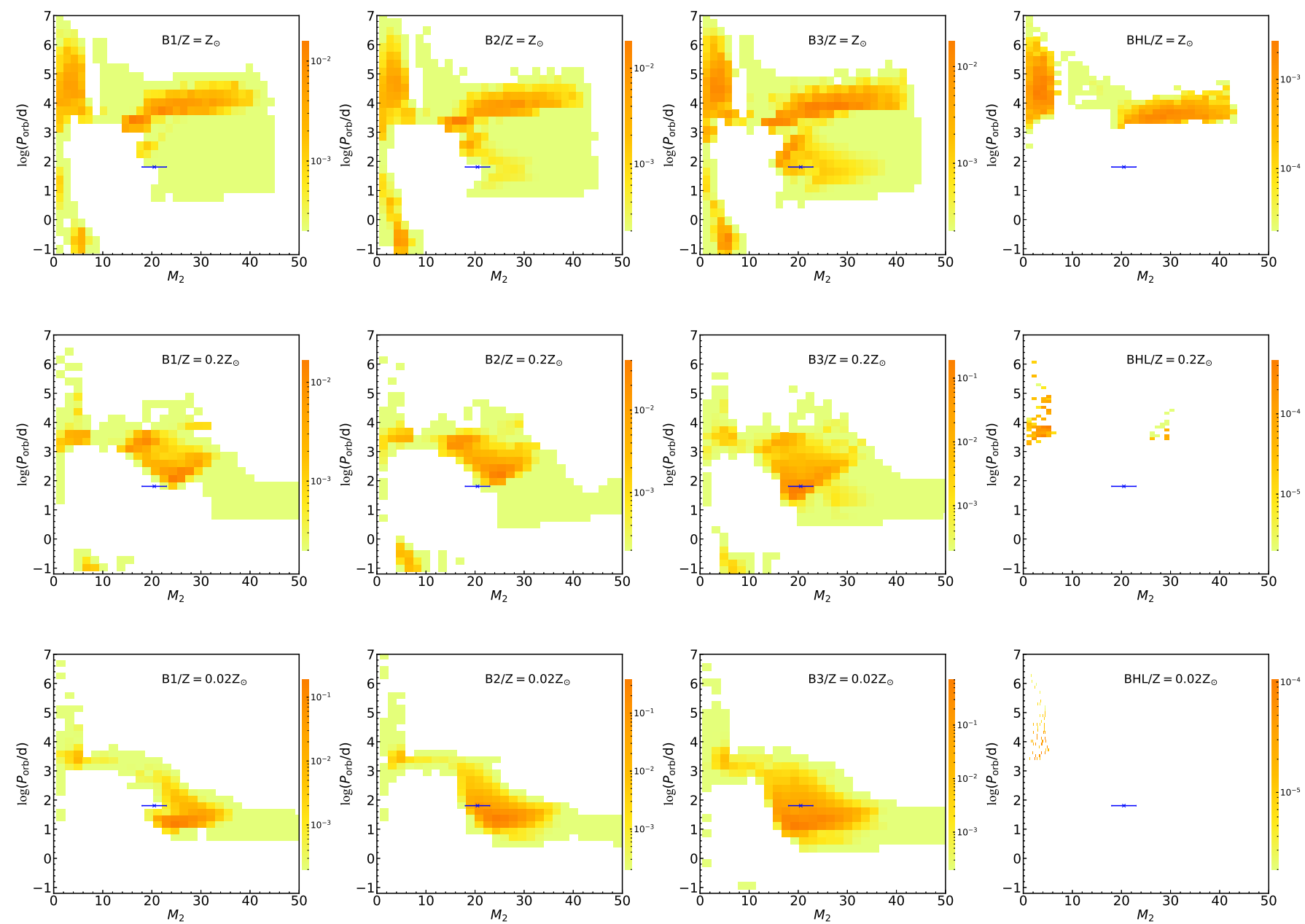

Fig. 1. Current orbital period $P_{\mathrm{orb}}-M_{2}$ distributions for models B1, B2, B3, and BHL at $Z=Z_{\odot}$ (upper panel), $0.2 Z_{\odot}$ (middle panel), and $0.02 Z_{\odot}$ (lower panel), respectively. The color represents the number of wind-fed NS ULXs (i.e., $L_{\mathrm{X}}>10^{39} \mathrm{erg} \mathrm{s}^{-1}$ ) in each matrix element. The cross with errorbars is the location of $\mathrm{P} 13$. The labels $\mathrm{B} 1, \mathrm{~B} 2, \mathrm{~B} 3$, and $\mathrm{BHL}$ in the panels represent WRLOF models with $\beta=1$ (B1), $\beta=2$ (B2), $\beta=3$ (B3), and BHL models, respectively. For example, the model B1/Z $=Z_{\odot}$ represents WRLOF model with $\beta=1$ (B1) and $Z=Z_{\odot}$.

Table 1. Expected number of wind-fed NS ULXs (i.e., $L_{\mathrm{X}}>$ $10^{39} \mathrm{erg} \mathrm{s}^{-1}$ ) for a Milky-Way-like galaxy with $\mathrm{SFR}=3 M_{\odot} \mathrm{yr}^{-1}$ for models B1, B2, B3, and $\mathrm{BHL}$ at $Z=Z_{\odot}, 0.2 Z_{\odot}$, and $0.02 Z_{\odot}$, respectively.

\begin{tabular}{lcccc}
\hline \hline & B1 & B2 & B3 & BHL \\
\hline$Z_{\odot}$ & $3.1 \times 10^{-1}$ & $3.7 \times 10^{-1}$ & $6.8 \times 10^{-1}$ & $9.0 \times 10^{-2}$ \\
\hline $0.2 Z_{\odot}$ & $3.2 \times 10^{-1}$ & $6.7 \times 10^{-1}$ & 1.5 & $7.0 \times 10^{-3}$ \\
\hline $0.02 Z_{\odot}$ & 1.1 & 2.9 & 6.3 & $2.3 \times 10^{-3}$ \\
\hline
\end{tabular}

\section{Results}

Our models show conclusively that with the WRLOF scenario taken into account, the population of wind-fed NS ULXs is distinct in number and binary parameters from that in the traditional BHL mode. Additionally, it is strongly metallicity dependent.

In Fig. 1, we show the distribution of current orbital period $P_{\text {orb }}$ and donor mass $M_{2}$ in models B1, B2, B3, and BHL, respectively. The upper, middle, and lower panels are for metallicities with $Z=Z_{\odot}$ (i.e., Solar), $Z=0.2 Z_{\odot}$, and $Z=0.02 Z_{\odot}$, respectively. The color represents the number of wind-fed NS ULXs (i.e., $L_{\mathrm{X}}>10^{39} \mathrm{erg} \mathrm{s}^{-1}$ ) in each matrix element. Overplotted is the PULX P13 (Fürst et al. 2016; Israel et al. 2017a), which has a $20 M_{\odot}$ B9Ia donor star in a $\sim 64$ day orbit (Motch et al. 2014). It is clear that the source P13 cannot be reproduced at all in any of the BHL cases, and the number of wind-fed NS ULXs in this case is low, especially in lower metallicities. However, when WRLOF is applied, many more NS ULXs are produced when comparing models B1, B2, and B3 with BHL models (see Table 1). The number of NS ULXs is enhanced greatly, about an order of magnitude in the solar case, and even more than two in lower metallicities. It is reasonable, as in the traditional BHL wind mode, wind accretion is much less efficient than RLOF, which has been neglected in previous studies (Linden et al. 2010; Shao \& Li 2015; Wiktorowicz et al. 2017). However in the WRLOF mode, the mass-accretion rates are significantly enhanced, sometimes by more than an order of magnitude (El Mellah et al. 2019a, see their Fig. 3), which helps to reach a typical ULX luminosity level. When compared with current observational statistics (i.e., $\sim 2$ ULXs per $M_{\odot} \mathrm{yr}^{-1}$ of SFR, Grimm et al. 2003; Swartz et al. 2011), our results are sound, that is, $\sim 0.1$ ULXs (at $Z=Z_{\odot}$ ) to $\sim 1 \mathrm{ULXs}\left(Z=0.02 Z_{\odot}\right)$ per $M_{\odot} \mathrm{yr}^{-1}$ of SFR. We suggest that the WRLOF scenario should be taken into account for the study of ULX and its populations in the future.

However the component of WRLOF NS ULXs between models (i.e., B1, B2, and B3, we do not discuss BHL models 

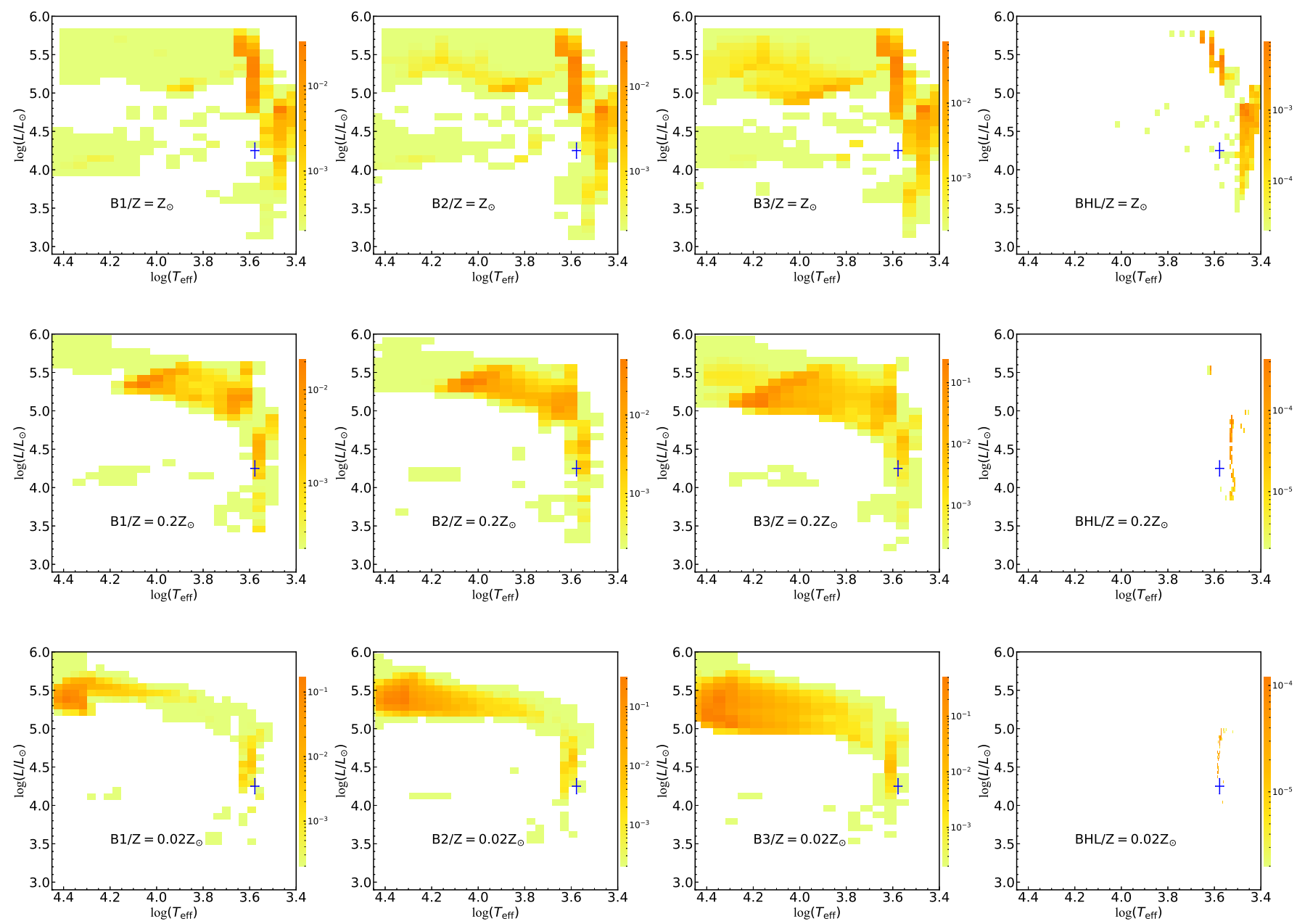

Fig. 2. Expected distributions of donor stars in $\mathrm{H}-\mathrm{R}$ diagram for models $\mathrm{B} 1, \mathrm{~B} 2, \mathrm{~B} 3$, and $\mathrm{BHL}$ at $Z=Z_{\odot}$ (upper panel), $0.2 Z_{\odot}$ (middle panel), and $0.02 Z_{\odot}$ (lower panel), respectively. The color represents the number of wind-fed NS ULXs (i.e., $L_{\mathrm{X}}>10^{39} \mathrm{erg} \mathrm{s}^{-1}$ ) in each matrix element. The cross is the location of the red supergiant donor star in PULX NGC 300 ULX-1. The labels B1, B2, B3, and BHL in the panels represent WRLOF models with $\beta=1$ (B1), $\beta=2$ (B2), $\beta=3$ (B3), and BHL models, respectively. For example, the model $\mathrm{B} 1 / Z=Z_{\odot}$ represents the WRLOF model with $\beta=1(\mathrm{~B} 1)$ and $Z=Z_{\odot}$.

hereafter due to their rarity) is distinct. The different species reveal that they may come from different evolutionary pathways. We note the most common subpopulation is sources with massive $\left(\sim 15-40 M_{\odot}\right)$ (super)giant donors in WRLOF models (i.e., B1, B2, and B3). But the distribution of current orbital period $P_{\text {orb }}$ is different and much shorter in lower metallicities $\left(\lesssim 10^{3}\right.$ days in model B3; $\lesssim 10^{4}$ days in model B2) when compared with that in the solar cases $\left(\leqslant 10^{5}\right.$ days in model B1). The phenomena we obtained here are very similar to that found by Linden et al. (2010) for supergiant high-mass X-ray binaries (SG-HMXBs, see the lower panel in their Fig. 5). The reason is that the donor with a poor metallicity is more compact than the rich ones (Linden et al. 2010; Klencki et al. 2020), particularly the maximum radius during the (sub)giant phase drops precipitously with decreasing metallicity (see their Fig. 7, Linden et al. 2010). So during the expansion of the donor before filling its RL, the orbit can be more compact at lower metallicities, while much wider at near solar metallicities. We note the donor of this population is mostly rejuvenated, that is, getting mass during the first RLOF from the more massive primary, which produces the NS after the SNe (age within $\sim 10 \mathrm{Myr}$ ). After that, the WRLOF phase may take place soon thereafter, lasting $\sim 0.1-1 \mathrm{Myr}$ before the donor fills its RL, after which a common envelope (CE) occurs due to a large donor-to-compact object mass ratio, resulting in a binary merger. Another subpopulation is sources with lower donor masses (i.e., $M_{2}<10 M_{\odot}$ ). Especially at solar metallicity (upper panel, models $\mathrm{B} 1 / Z=Z_{\odot}$, $\mathrm{B} 2 / Z=Z_{\odot}$, and $\mathrm{B} 3 / Z=Z_{\odot}$ ), it clearly contains two species: one being sources with longer orbital periods (i.e., $P_{\text {orb }}>100$ days), which mainly come from binaries with a less massive progenitor $\left(<10 M_{\odot}\right)$, and large initial separations $\left(\sim 10^{3}-10^{4} R_{\odot}\right)$. We note that when the star formation history is shortened to within tens of million years, corresponding to intense star burst cases, this population would drop. The other species is sources with short orbital periods (i.e., $P_{\text {orb }} \sim 0.1-100$ days). We note that they are mainly stripped He-rich stars, which are produced from a CE.

In addition, we find that there is a trend that the larger the value of $\beta$, the more WRLOF NS ULXs are produced in each metallicity case. The reason is mainly related to the dependence of $\mu$ (i.e., the fraction of stellar wind captured by the accretor) on the exponent $\beta$ of the velocity law, as illustrated in Fig. 2 by El Mellah et al. (2019a). It is clear in the figure that the higher $\beta$ is, the larger $\mu$ is in the same condition, as the higher $\beta$, the later $v_{\infty}$ is matched, hence the larger $\mu$ as seen in their Fig. 2 and stated by El Mellah et al. (2019a). Furthermore, it is notable that P13 can be produced in all metallicity cases as well, especially in 
cases with larger values of $\beta$ and lower metallicities. Additionally, there is a weak trend that more WRLOF NS ULXs seem to be produced in lower metallicities, when compared with the solar cases, which is similar to the finding recently obtained by Marchant et al. (2017). It is because of the increase in $\mu$ with decreasing $\eta$, which is the most significant effect shown in their Fig. 2. We note sources in lower metallicities are more compact, that is, a smaller $P_{\text {orb }}$, then a larger $v_{\text {orb }}=2 \pi a / P_{\text {orb }}$, hence a smaller $\eta=v_{\infty} / v_{\text {orb }}$, which gives a larger $\mu$, resulting in more NS ULXs instead, although the wind mass loss is relatively lower in the low-metallicity regime.

The donor of ULXs is the key to understanding the nature of ULXs and the population. In Fig. 2, we present the expected distributions of the luminosity $\log \left(L_{\mathrm{bol}} / L_{\odot}\right)$ and effective temperature $\log \left(T_{\mathrm{eff}} / \mathrm{K}\right)$ of donor stars (i.e., H-R diagram) for models B1, B2, B3, and BHL, at $Z=Z_{\odot}$ (i.e., Solar, upper panel), $Z=0.2 Z_{\odot}$ (middle panel), and $Z=0.02 Z_{\odot}$ (lower panel), respectively. The color represents the number of windfed NS ULXs (i.e., $L_{X}>10^{39} \mathrm{erg} \mathrm{s}^{-1}$ ) in each matrix element. The cross is the location of PULX NGC 300 ULX-1 (Carpano et al. 2018; Heida et al. 2019b) with a donor counterpart recently discovered as a RSG star $\left(T_{\text {eff }}=3650-3900 \mathrm{~K}\right.$ and $\log \left(L_{\text {bol }} / L_{\odot}\right)=4.25 \pm 0.10$, Heida et al. 2019a). It is clear that in each metallicity case, the source can be reproduced roughly in all WRLOF models. However, the percentage of RSG in all (super)giant donors changes largely as metallicity changes. It dominates in near solar metallicities (upper panel), but declines significantly as metallicity decreases. Also there is less of it in very low metallicity (i.e., $Z=0.02 Z_{\odot}$, lower panel). We note that RSG NS ULXs usually have lower donor masses (i.e., $M_{2}<10 M_{\odot}$ ) with longer orbital periods (i.e., $P_{\text {orb }}>100$ days), while other (super)giant NS ULXs mainly have massive donors $\left(\sim 15-40 M_{\odot}\right)$, which are hotter and brighter, as expected.

\section{Discussion and concluding remarks}

This study shows that with the WRLOF accretion mode taken into account, it is possible to model the population of (super)giant NS ULXs, although the results are still subject to some uncertainties and simplified treatments. For example, the stellar wind of massive stars is vital in our simulations; however, it is still highly uncertain (Puls et al. 2008; Sander \& Vink 2020), in particular due to the effects of clumping, other unsteady modes of mass loss, and the effects caused by a binary among massive stars (Smith 2014), about which our knowledge is still poor. In this study, we adopted a simplified parameter, $\beta_{\text {wind }}$, to depict the velocity of stellar wind. Normally, a smaller $\beta_{\text {wind }}$ means a smaller wind velocity $v_{\infty}$, hence a smaller $\eta=v_{\infty} / v_{\text {orb }}$, resulting in a higher mass transfer efficiency $\mu$. We also varied the value of $\beta_{\text {wind }}$ for very hot stars from 7 to 3 , but we find no significant changes in our results. Additionally, for the super-Eddington accretion, geometrical beaming may take place (King et al. 2001; Poutanen et al. 2007; King 2008; King \& Lasota 2020), but it is still very uncertain (Abarca et al. 2018; Mushtukov et al. 2021). We also tested this scenario and find that our conclusion is largely unchanged. Finally, we note that the absolute formation rate of (wind-fed) NS ULXs is highly uncertain. Several parameters, such as the star formation rate and history of the galaxy, the binary fraction, the IMF of the primary and secondary stars, and the natal kick of newborn NS (Zuo et al. 2014) may affect it significantly by up to one or two orders of magnitude. Unique galaxies such as ring galaxies (Wolter et al. 2018) are helpful to further address this issue (i.e., the relative and absolute formation rate of different kinds of ULXs through the X-ray luminosity function modeling, see Zuo et al. 2014, for example), which is in preparation, however, and beyond the scope of this Letter.

Nevertheless, this study still shows that the population of wind-fed NS ULXs in WRLOF mode is distinct in number and the binary parameters from that in the traditional BHL mode, and it is strongly metallicity dependent. The number of NS ULXs with (super)giant donors is enhanced greatly by about an order of magnitude in solar cases, and even more than two in lower metallicities (see Table 1) when considering the WRLOF accretion mode. Furthermore, they are heavily metallicity-dependent. The most common population in all WRLOF models is sources with massive $\left(\sim 15-40 M_{\odot}\right)$ (super)giant donors, which dominate in lower metallicities. However, that is not the case in the solar case, which is dominated by RSG with lower mass $M_{2}<10 M_{\odot}$ instead. Moreover, the two NS ULXs (i.e., P13 and NGC 300 ULX-1) can be well reproduced in the WRLOF paradigm, which significantly enriches our understanding of the nature of ULXs and the population. We also present the current distributions of binary parameters (see Figs. 1 and 2), which may be further testified by high-resolution optical and X-ray observations of (NS)ULXs populations in the future.

Acknowledgements. We thank Ileyk El Mellah for providing the data tables that help to conduct the simulation. We are grateful to Ileyk El Mellah for useful discussions on the WRLOF scheme and Jorick S. Vink for the constructive suggestions on the wind velocity of He-rich star. This work is supported by the National Natural Science Foundation of China (grant Nos. 11573021, U1938104, 12003020) and the Fundamental Research Funds for the Central Universities.

\section{References}

Abarca, D., Kluźniak, W., \& Sądowski, A. 2018, MNRAS, 479, 3936 Bachetti, M., Harrison, F. A., Walton, D. J., et al. 2014, Nature, 514, 202 Begelman, M. C. 2002, ApJ, 568, L97 Belczynski, K., Kalogera, V., Rasio, F. A., et al. 2008, ApJS, 174, 223 Belczynski, K., Bulik, T., Fryer, C. L., et al. 2010, ApJ, 714, 1217 Belczynski, K., Heger, A., Gladysz, W., et al. 2016, A\&A, 594, A97 Bondi, H., \& Hoyle, F. 1944, MNRAS, 104, 273

Brightman, M., Harrison, F. A., Fürst, F., et al. 2018, Nat. Astron., 2, 312 Carpano, S., Haberl, F., Maitra, C., \& Vasilopoulos, G. 2018, MNRAS, 476, L45 Colbert, E. J. M., \& Mushotzky, R. F. 1999, ApJ, 519, 89

Dall'Osso, S., Perna, R., Papitto, A., Bozzo, E., \& Stella, L. 2016, MNRAS, 457, 3076

Dessart, L., Burrows, A., Ott, C. D., et al. 2006, ApJ, 644, 1063

Eggleton, P. P. 1983, ApJ, 268, 368

Ekşi, K. Y., Andaç, İ. C., Çıkıntoğlu, S., et al. 2015, MNRAS, 448, L40

El Mellah, I., Sundqvist, J. O., \& Keppens, R. 2019a, A\&A, 622, L3

El Mellah, I., Sander, A. A. C., Sundqvist, J. O., \& Keppens, R. 2019b, A\&A, 622, A189

Fabbiano, G. 1989, ARA\&A, 27, 87

Fragos, T., Linden, T., Kalogera, V., \& Sklias, P. 2015, ApJ, 802, L5

Fryer, C. L., Belczynski, K., Wiktorowicz, G., et al. 2012, ApJ, 749, 91

Fürst, F., Walton, D. J., Harrison, F. A., et al. 2016, ApJ, 831, L14

Fürst, F., Walton, D. J., Heida, M., et al. 2018, A\&A, 616, A186

Gladstone, J. C., Roberts, T. P., \& Done, C. 2009, MNRAS, 397, 1836

Grimm, H. J., Gilfanov, M., \& Sunyaev, R. 2003, MNRAS, 339, 793

Hamann, W. R., \& Koesterke, L. 1998, A\&A, 335, 1003

Heida, M., Jonker, P. G., Torres, M. A. P., et al. 2014, MNRAS, 442, 1054

Heida, M., Torres, M. A. P., Jonker, P. G., et al. 2015, MNRAS, 453, 3511

Heida, M., Jonker, P. G., Torres, M. A., et al. 2016, MNRAS, 459, 771

Heida, M., Harrison, F. A., Brightman, M., et al. 2019a, ApJ, 871, 231

Heida, M., Lau, R. M., Davies, B., et al. 2019b, ApJ, 883, L34

Hobbs, G., Lorimer, D. R., Lyne, A. G., \& Kramer, M. 2005, MNRAS, 360, 974

Humphreys, R. M., \& Davidson, K. 1994, PASP, 106, 1025

Hurley, J. R., Pols, O. R., \& Tout, C. A. 2000, MNRAS, 315, 543

Hurley, J. R., Tout, C. A., \& Pols, O. R. 2002, MNRAS, 329, 897

Israel, G. L., Papitto, A., Esposito, P., et al. 2017a, MNRAS, 466, L48

Israel, G. L., Belfiore, A., Stella, L., et al. 2017b, Science, 355, 817

Kaaret, P., Ward, M. J., \& Zezas, A. 2004, MNRAS, 351, L83

Kaaret, P., Feng, H., \& Roberts, T. 2017, ARA\&A, 55, 303

King, A. R. 2008, MNRAS, 385, L113 
King, A., \& Lasota, J.-P. 2020, MNRAS, 494, 3611

King, A. R., Davies, M. B., Ward, M. J., Fabbiano, G., \& Elvis, M. 2001, ApJ, 552, L109

Klencki, J., Nelemans, G., Istrate, A. G., \& Pols, O. 2020, A\&A, 638, A55

Kroupa, P., Tout, C. A., \& Gilmore, G. 1993, MNRAS, 262, 545

Lamers, H. J. G. L. M., Snow, T. P., \& Lindholm, D. M. 1995, ApJ, 455, 269

Lau, R. M., Heida, M., Walton, D. J., et al. 2019, ApJ, 878, 71

Linden, T., Kalogera, V., Sepinsky, J. F., et al. 2010, ApJ, 725, 1984

Liu, J., Bregman, J. N., \& Seitzer, P. 2004, ApJ, 602, 249

Liu, J.-F., Bregman, J. N., Bai, Y., Justham, S., \& Crowther, P. 2013, Nature, 503, 500

Loveridge, A. J., van der Sluys, M. V., \& Kalogera, V. 2011, ApJ, 743, 49

Marchant, P., Langer, N., Podsiadlowski, P., et al. 2017, A\&A, 604, A55

Mohamed, S., \& Podsiadlowski, P. 2007, in 15th European Workshop on White Dwarfs, eds. R. Napiwotzki, \& M. R. Burleigh, ASP Conf. Ser., 372, 397

Motch, C., Pakull, M. W., Soria, R., Grisé, F., \& Pietrzyński, G. 2014, Nature, 514,198

Mushtukov, A. A., Portegies Zwart, S., Tsygankov, S. S., Nagirner, D. I., \& Poutanen, J. 2021, MNRAS, 501, 2424

Orosz, J. A., McClintock, J. E., Aufdenberg, J. P., et al. 2011, ApJ, 742, 84

Podsiadlowski, P., \& Mohamed, S. 2007, Balt. Astron., 16, 26

Podsiadlowski, P., Langer, N., Poelarends, A. J. T., et al. 2004, ApJ, 612, 1044

Poutanen, J., Lipunova, G., Fabrika, S., Butkevich, A. G., \& Abolmasov, P. 2007, MNRAS, 377, 1187
Puls, J., Vink, J. S., \& Najarro, F. 2008, A\&ARv, 16, 209

Rodríguez Castillo, G. A., Israel, G. L., Belfiore, A., et al. 2020, ApJ, 895, 60 Sander, A. A. C., \& Vink, J. S. 2020, MNRAS, 499, 873

Sathyaprakash, R., Roberts, T. P., Walton, D. J., et al. 2019, MNRAS, 488, L35 Shao, Y., \& Li, X. D. 2015, ApJ, 802, 131

Smith, N. 2014, ARA\&A, 52, 487

Sutton, A. D., Roberts, T. P., \& Middleton, M. J. 2013, MNRAS, 435, 1758

Swartz, D. A., Soria, R., Tennant, A. F., \& Yukita, M. 2011, ApJ, 741, 49

Tong, H. 2015, Res. Astron. Astrophys., 15, 517

Villar, V. A., Berger, E., Chornock, R., et al. 2016, ApJ, 830, 11

Vink, J. S., \& de Koter, A. 2005, A\&A, 442, 587

Vink, J. S., de Koter, A., \& Lamers, H. J. G. L. M. 2001, A\&A, 369, 574

Walton, D. J., Fürst, F., Heida, M., et al. 2018, ApJ, 856, 128

Webbink, R. F. 1984, ApJ, 277, 355

Webbink, R. F. 2008, in Common Envelope Evolution Redux, eds. E. F. Milone, D. A. Leahy, \& D. W. Hobill, 352, 233

Wiktorowicz, G., Sobolewska, M., Sądowski, A., \& Belczynski, K. 2015, ApJ, 810,20

Wiktorowicz, G., Sobolewska, M., Lasota, J.-P., \& Belczynski, K. 2017, ApJ, 846,17

Wiktorowicz, G., Lasota, J.-P., Middleton, M., \& Belczynski, K. 2019, ApJ, 875, 53

Wolter, A., Fruscione, A., \& Mapelli, M. 2018, ApJ, 863, 43

Xu, X. J., \& Li, X. D. 2010, ApJ, 716, 114

Zuo, Z.-Y., \& Li, X.-D. 2014, MNRAS, 442, 1980

Zuo, Z.-Y., Li, X.-D., \& Gu, Q.-S. 2014, MNRAS, 437, 1187 